\title{
Cambios ontogénicos en la morfología de plántulas de Manilkara zapota: análisis de sus implicaciones ecológicas
}

\author{
Ontogenetic changes in seedling morphology of Manilkara zapota: analysis of ecological \\ implications
}

\author{
Juan Antonio Cruz-Rodríguez y Lauro López-Mata ${ }^{2 *}$ \\ ${ }^{1}$ Departamento de Agroecología, Universidad Autónoma Chapingo. Texcoco 56230, Estado de México, México. ${ }^{2}$ Programa de Botánica, Colegio de \\ Postgraduados. 56230, Montecillo, Texcoco Estado de México, México. \\ *Correspondencia: lauro@colpos.mx
}

\begin{abstract}
Resumen. En este trabajo se describen los cambios ontogénicos que se presentan en la morfología de plántulas de Manilkara zapota, uno de los árboles más abundantes de las selvas neotropicales. Estas plántulas presentan cambios morfológicos que se relacionan significativamente con sus probabilidades de supervivencia. La variación se describe a partir del registro y la observación de 552 plántulas con alturas menores a $35 \mathrm{~cm}$, localizadas en una selva mediana subperennifolia en la parte central del estado de Veracruz. De cada individuo se registró presencia de cotiledones, altura, número de hojas, longitud y forma de las hojas, cobertura y área foliar específica (AFE). Con base en la forma de las hojas, se distinguieron 4 variantes: a) plántulas con cotiledones; b) plántulas con hojas elípticas; $c$ ) plántulas con hojas oblongas, y d) plántulas con hojas oblongolanceoladas. El tamaño de las hojas de la última clase fue de hasta $30 \mathrm{~cm}$ y duplica el tamaño de las hojas de los árboles adultos. El AFE mostró una correlación negativa con el tamaño de la hoja, lo que sugiere costos de construcción altos en las hojas grandes y explica su ausencia en los primeros años de vida. La formación de hojas grandes es un proceso discontinuo que depende de las reservas de la plántula y que permite aprovechar la escasa radiación prevaleciente en sotobosque.
\end{abstract}

Palabras clave: selva mediana subperennifolia, supervivencia y crecimiento de plántulas, especies tolerantes a la sombra, área foliar específica.

\begin{abstract}
In this work the variability in seedling morphology of one of the most abundant neotropical trees, Manilkara zapota, is described. These seedlings display morphological changes significantly related with their survival probabilities. The variation is described from observations of 552 seedlings with heights less than $35 \mathrm{~cm}$, in a subtropical rain forest in the central portion of the state of Veracruz. From each seedling we gathered the following data: presence of cotyledons, height, number of leaves, length and shape of the leaves, cover, and specific leaf area (SLA). Based on leaf shape, 4 seedling variants were distinguished: a) seedlings with cotyledons, b) seedlings with elliptic leaves, c) seedlings with oblong leaves and, d) seedlings with oblong-lanceolated leave. The leaf size of the last-mentioned variant was of up to $30 \mathrm{~cm}$ length, which duplicates the size of the mature trees leaves. SLA showed a negative correlation with leaf size suggesting a high construction costs for large leaves which explains their absence in the first years of life. The development of large leaves is a non-continuous process that depends on the seedling reserves allowing them to take advantage of the scarce light in the understory.
\end{abstract}

Kew words: subtropical rain forest, seedling growth and survival, shade tolerant species, specific leaf area.

\section{Introducción}

Los árboles que alcanzan el dosel de las selvas tropicales experimentan, a lo largo de su ontogenia, cambios significativos en la disponibilidad de luz (Rozendaal et al., 2006). Inician su desarrollo en el sotobosque con el $1-2 \%$ de la radiación que llega a la parte superior del

Recibido: 14 agosto 2007; aceptado: 04 septiembre 2009 dosel y reciben grandes cantidades de luz al abrirse un claro o al superar en altura a los otros árboles (Chazdon, 1988). Para responder a estas variaciones las plantas deben poseer la capacidad de ajustarse a cambios de diferente magnitud y distintas escalas de tiempo (Sterk et al., 1999). Las respuestas pueden ser fisiológicas, relacionadas con el balance hídrico y con las tasas de fotosíntesis (Baltzer y Thomas, 2007; Rijkers et al., 2000), morfológicas, sobre todo a nivel de hojas (Agyeman et al., 1999) o de 
arquitectura de la planta (Kohyama, 1987; Poorter, 1999). La capacidad de ajuste a la variación lumínica influye en el crecimiento y supervivencia de las plantas, aunque los mecanismos desarrollados muestran diferencias significativas entre especies (Sterck et al., 2003; Kitajima, 1994).

Los ajustes observados pueden ser el resultado de procesos de aclimatación (Rozendaal et al., 2006) o pueden ser cambios inherentes a las especies, es decir ontogénicos, que dependen en gran medida del tamaño de los individuos (Poorter, 1999; Ackerly, 1997; Thomas e Ickes, 1995). Una idea ampliamente difundida establece que los individuos juveniles de árboles tropicales producen hojas más grandes que los adultos de la misma especie (Thomas e Ickes, 1995; Whitmore, 1984). A pesar de que no es posible generalizar esta idea, en algunos grupos de especies, sobre todo los que alcanzan el dosel de la selva, hay una clara dirección en el tamaño de las hojas conforme se avanza en la ontogenia. El incremento en el tamaño de las hojas se inicia desde la etapa de plántulas y alcanza un máximo antes de que los árboles entren en etapa reproductiva (Thomas e Ickes, 1995).

En el caso del árbol tropical Manilkara zapota L. (Royen) (Sapotacea), que es una de las especies que dominan el dosel de las selvas altas y medianas de México y Centroamérica, los individuos que se desarrollan bajo condiciones de dosel cerrado, con el tiempo transitan de una condición en la que sólo presentan cotiledones a otra en la que gradualmente desarrollan hojas de mayor longitud. El incremento en el tamaño de las hojas conlleva aumentos significativos en el tiempo de supervivencia en las plántulas de esta especie (Cruz- Rodríguez y LópezMata, 2004).

Las descripciones de la morfología de las plántulas y sus cambios ontogénicos no son comunes y con frecuencia se circunscriben a la morfología que presentan las plántulas poco después de la germinación, por lo que se ha puesto especial énfasis en la forma, tamaño y posición de los cotiledones (Ng, 1978; Miquel, 1987). No obstante, la literatura sobre la ecología de plántulas de árboles tropicales incluye información de individuos recién germinados (Garwood, 1996) y de individuos de hasta un metro de altura (Turner, 1990). Esta situación dificulta las comparaciones ya que es obvio que los procesos demográficos y las condiciones a las que se encuentran sometidos estos individuos son significativamente diferentes (Garwood, 1996). En el caso de Manilkarazapota las descripciones de la especie se han limitado a la etapa adulta (Pennington, 1992; Pennigton y Sarukhán, 2004), sin embargo, el conocimiento de las etapas tempranas de la ontogenia es fundamental para comprender la dinámica poblacional de la especie (Cruz-Rodríguez et al., 2009).
En este trabajo se hace una descripción morfológica de las plántulas de $M$. zapota y se compara la morfología de sus hojas con la de las hojas de los individuos adultos. Se presenta una clasificación y se discuten las posibles implicaciones ecológicas de los cambios en la ontogenia.

\section{Materiales y métodos}

La evaluación de las características morfológicas de las plántulas de $M$. zapota se realizó en un grupo de 552 individuos, localizados en 8 cuadros de $10 \mathrm{~m}^{2}(5 \mathrm{~m} \times 2 \mathrm{~m})$, establecidos en el sotobosque de una selva mediana subperennifolia (sensu Miranda y Hernández-X., 1963).

Esta selva está dominada por Brosimum alicastrum Sw. (Moraceae) y M. zapota, y ha sido excluida de la explotación forestal desde hace más de 100 años (GodínezIbarra y López-Mata, 2002). El sitio se localiza en la zona de Protección de Flora y Fauna Santa Gertrudis (entre las coordenadas $19^{\circ} 49^{\prime} 37^{\prime \prime}$ y 19 $19^{\circ} 51^{\prime} 50^{\prime \prime} \mathrm{N}$ y los $96^{\circ} 32^{\prime} 37^{\prime \prime}$

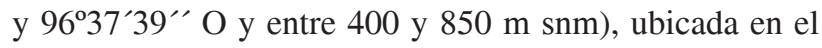
municipio de Vega de Alatorre, Veracruz, México. Para cada individuo se registraron las siguientes variables: $a$ ) presencia de cotiledones; b) altura $(\mathrm{cm})$; c) número de hojas; e) longitud (cm) de la hoja más larga (LHML), y d) cobertura $\left(\mathrm{cm}^{2}\right)$. Sólo se midió la longitud de la hoja más grande, ya que una plántula puede tener hojas de diferente tamaño, encontrándose las más pequeñas en la base y las más largas en el ápice. La cobertura de las plántulas se calculó como:

$$
\left(\pi \frac{D_{1} \times D_{2}}{4}\right)
$$

donde $\mathrm{D}_{1}$ y $\mathrm{D}_{2}$ son los valores de largo y ancho de la plántula, medidas en forma perpendicular.

Para entender el significado funcional de las hojas de distinto tamaño, se cálculo el área foliar específica (AFE) definida como el área de la hoja/peso seco de la hoja $\left(\mathrm{cm}^{2}\right.$ $\left.\mathrm{g}^{-1}\right)$. El AFE permite evaluar la capacidad que tienen las plantas para explotar ambientes con diferentes niveles de recursos. Valores altos de AFE se relacionan con hojas altamente productivas, de vida corta, vulnerables a los herbívoros y que se desempeñan mejor en ambientes ricos en recursos. En contraste, hojas con AFE baja funcionan mejor en ambientes pobres en recursos donde la retención de los recursos previamente capturados es la prioridad (Wilson et al., 1999).

El AFE se evaluó en una muestra de 40 individuos colectados en los 8 cuadros en la que se incluyeron plántulas de los diferentes estados. Se determinó el área foliar de cada uno $\left(\mathrm{cm}^{2}\right)$ mediante un integrador de área 
foliar LICOR modelo LI-3100, y el peso seco (g) de sus hojas (secadas a $70^{\circ} \mathrm{C}$ por 72 horas y pesadas en una balanza analítica).

\section{Resultados}

Con base en su morfología y en las características de sus hojas, las plántulas de M. zapota pueden ubicarse en 4 estados. El primero (a) lo conforman individuos con una altura promedio de $7.6 \pm 1.2 \mathrm{~cm}$ y que presentan sólo cotiledones. Los cotiledones son epigeos, foliáceos, delgados, de color verde oscuro y con una longitud de 34 centímetros, por lo que su morfología corresponde al tipo fanerocotilar epigeo foliáceo de Miquel (1987; Fig. 1a). Los cotiledones son muy persistentes y las plántulas son capaces de sobrevivir un promedio de 8 meses sin desarrollar hojas.

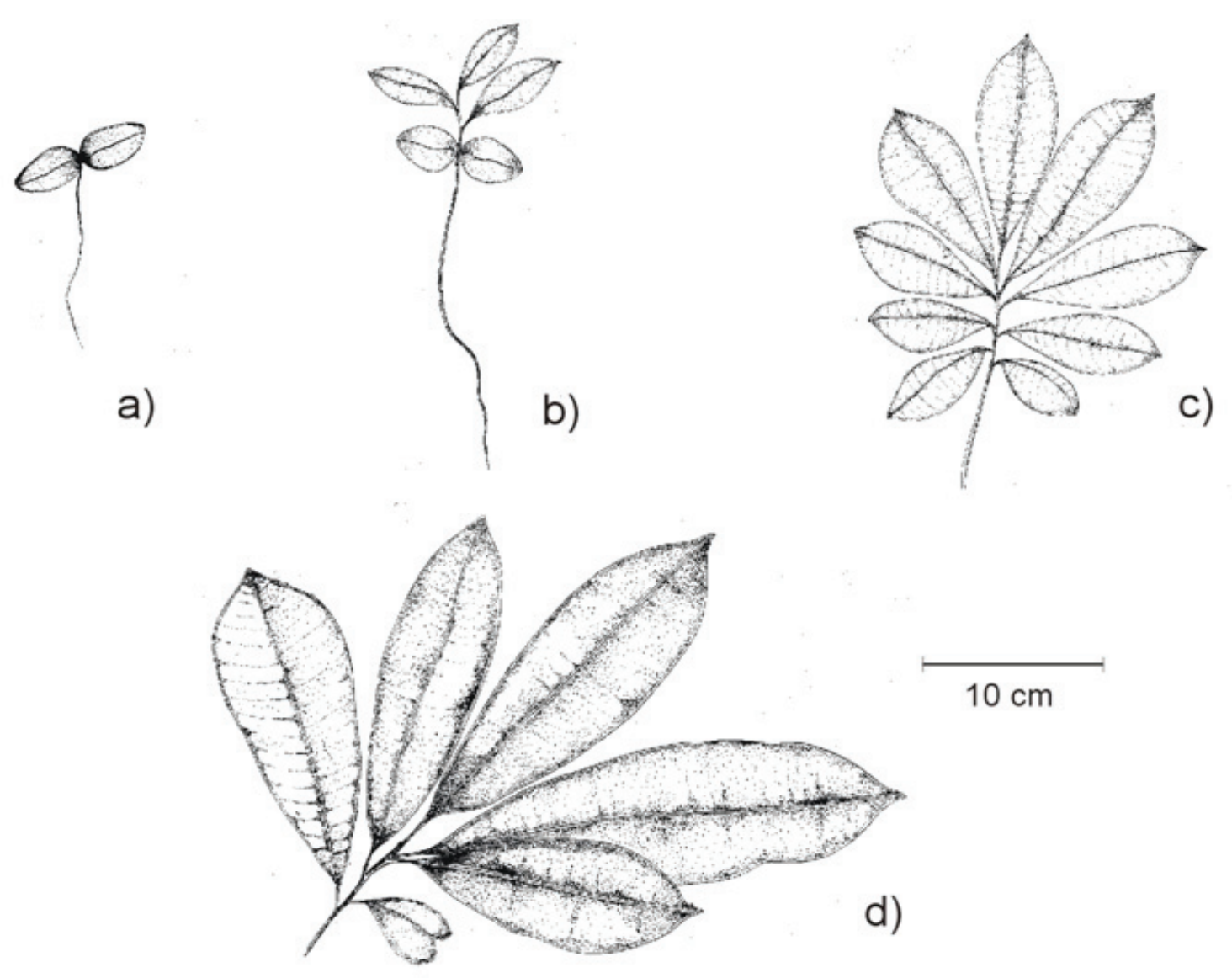

a)

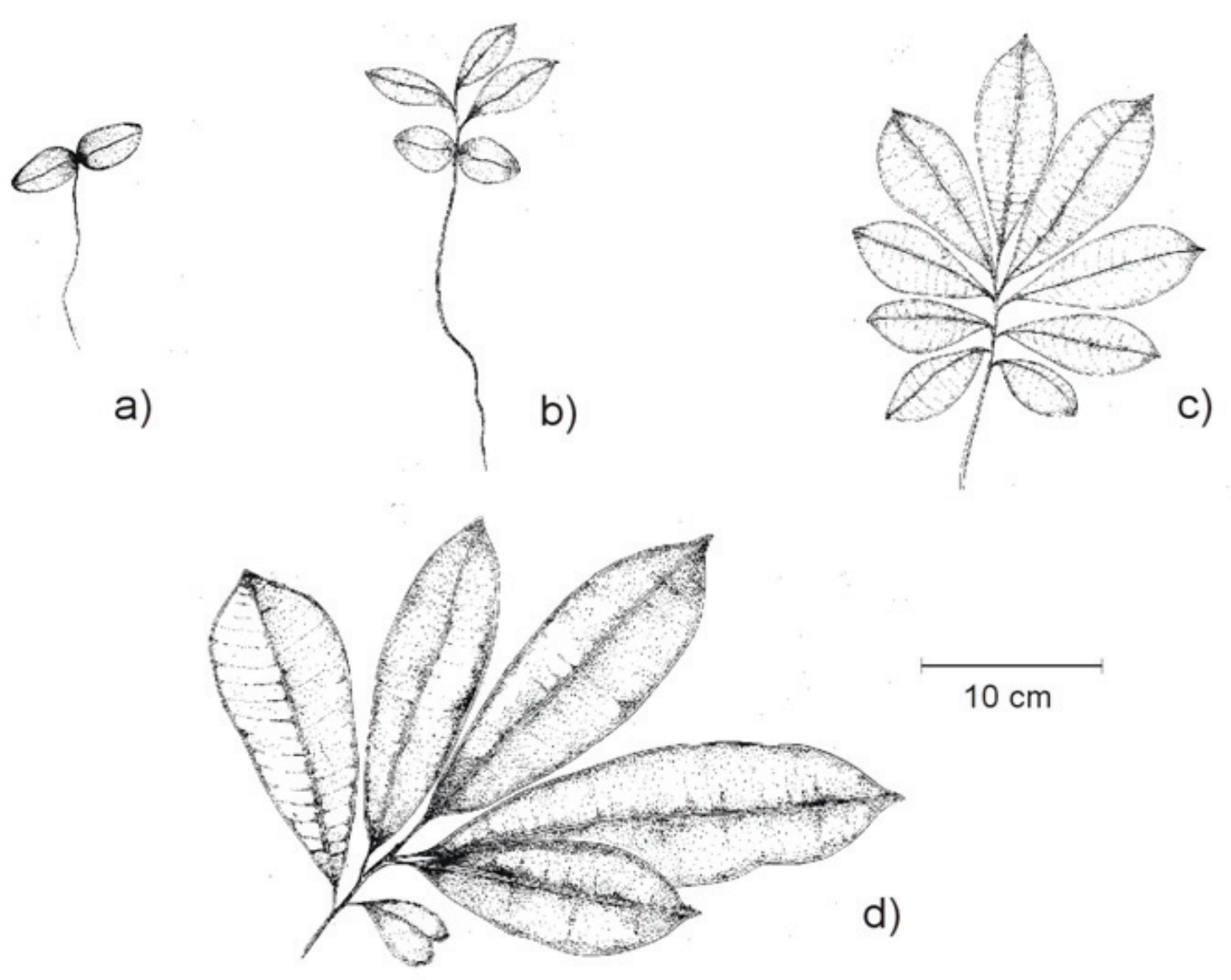

b)
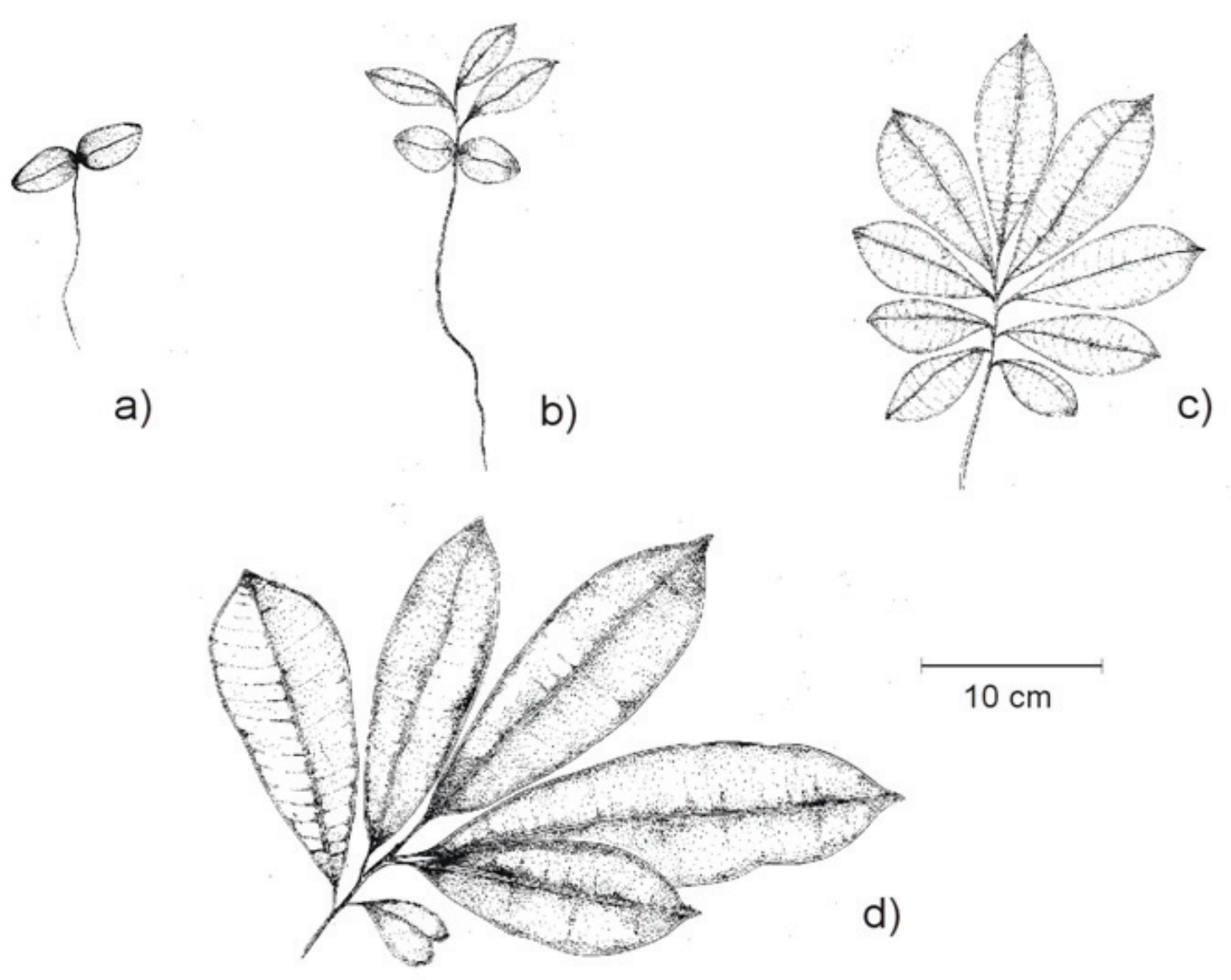
clasificarse en los otros 3 estados: plántulas con hojas elípticas (b); plántulas con hojas oblongas (c), y plántulas con hojas oblongolanceoladas (d).

Las hojas del segundo estado (b) tienen una longitud de 2 a $5 \mathrm{~cm}$ y una anchura de $1.5 \mathrm{~cm}$; su ápice es agudo o cortamente acuminado y poseen el margen entero. Presentan una disposición en espiral y se aglomeran en la parte superior del tallo; la altura de estos individuos fluctúa entre 10 y 15 centímetros.

Las plántulas del tercer estado (c), que tienen una altura entre 15 y $20 \mathrm{~cm}$, tienen hojas con láminas de 5 a $20 \mathrm{~cm}$ de largo y de 2 a $7 \mathrm{~cm}$ de ancho. El ápice de la hoja puede ser acuminado pero es común encontrar hojas con ápices obtusos o romos; casi en todas las hojas la base es aguda aunque en algunos individuos puede ser redondeada.

El cuarto estado (d) lo conforman plántulas de hojas oblongolanceoladas, es decir, con láminas todavía más

Figura 1. Morfología de las hojas de plántulas de M. zapota. a) plántula con cotiledones del tipo fanerocotilar epigeo foliáceo (FEF), según la clasificación de Miquel (1987); b) plántula con hojas elípticas que aún conserva sus cotiledones; c) plántula con hojas oblongas (porción de la parte aérea) y c) plántula cuyas hojas más grandes son oblongolanceoladas (porción de la parte aérea). La definición de la forma de la hoja de b, c y d con base en Font Quer (1977). Los individuos ubicados en la categoría de plántulas tienen alturas menores a los $35 \mathrm{~cm}$. 
largas que anchas, o largamente lanceoladas sensu Font Quer (1977). Su longitud puede ser hasta de $30 \mathrm{~cm}$ y su anchura no es mayor a $7 \mathrm{~cm}$; los ápices pueden ser tanto agudos como obtusos, aunque son más comunes los primeros. Las plántulas con este tipo de hojas presentan entrenudos cortos, con una gran cantidad de cicatrices foliares y una altura que varía entre 25 y 35 centímetros.

Las hojas oblongo lanceoladas con longitudes hasta de $30 \mathrm{~cm}$ se presentan en los individuos juveniles y su tamaño contrasta con el tamaño de las hojas de los individuos adultos cuya longitud promedio $( \pm$ d. s.) es de $15 \pm 2.3$ $\mathrm{cm}(\mathrm{n}=50)$.

El desarrollo de las plántulas conlleva una mayor superficie fotosintética producto del incremento, altamente significativo ( $\mathrm{p}<0.0001$ ), en el número de hojas, en el tamaño de las hojas y en la cobertura de la copa (Cuadro1). El peso mayor de este incremento recae en el aumento, en varios órdenes de magnitud, del promedio de la cobertura de la copa, que cambia de $43.03 \mathrm{~cm}^{2}$ en el segundo estado a $191.28 \mathrm{~cm}^{2}$ en el tercero y aumenta hasta 1097 $\mathrm{cm}^{2}$ en el último. Este incremento se explica sobre todo por desarrollo de hojas significativamente más grandes, ya que el cambio en el número promedio de hojas, a pesar de ser significativo $(\mathrm{p}<0.0001)$, sólo implicó un incremento de 3.75 a 8.6 hojas entre el segundo y el cuarto estado (Cuadro 1).

A pesar de que la altura cambia significativamente entre estados $(\mathrm{p}<0.0001)$ y de que existe una correlación positiva y significativa con la longitud de las hojas $(r=0.80$; $\mathrm{n}=552 ; \mathrm{p} \leq 0.05)$, la presencia de hojas cada vez más grandes no sólo es consecuencia del aumento en la altura de las plántulas. Si se compara la razón que se obtiene de dividir la altura de cada plántula entre la longitud de su hoja más grande, se observa que las plántulas con hojas elípticas poseen en promedio una altura 2.42 veces mayor que la longitud de sus hojas; las que poseen hojas oblongas tienen una razón promedio de 1.53 y las oblongolanceoladas de 1.03, ya que en este último caso las hojas tienen una longitud que es igual o mayor a la altura de los individuos (Cuadro1).

El AFE promedio ( \pm d. s.) para todas las plántulas fue de $14.5 \pm 2.7 \mathrm{~m}^{2} \mathrm{~kg}^{-1}$ y mostró una correlación negativa con el tamaño de la hoja $(\mathrm{r}=-0.56 ; \mathrm{n}=23$; $\mathrm{p} \leq 0.05)$; la comparación entre los 3 estados ontogénicos muestra diferencias estadísticamente significativas $(\mathrm{p}<0.01)$. Las hojas oblongolanceoladas $(n=10)$ presentan un promedio de AFE de $11.56 \pm 0.9 \mathrm{~m}^{2} \mathrm{~kg}^{-1}$, que es estadísticamente menor $(\mathrm{p}<0.001)$ al promedio del AFE tanto de las hojas oblongas $(\mathrm{n}=10), 18.25 \pm 8.4 \mathrm{~m}^{2} \mathrm{~kg}^{-1}$ como al de las hojas elípticas $(\mathrm{n}=20), 16.19 \pm 3.5 \mathrm{~m}^{2} \mathrm{~kg}^{-1}$; sin embargo, la diferencia en el AFE de estos 2 últimos casos no fue significativa $(\mathrm{p}>0.05)$.

\section{Discusión}

Las plántulas de los árboles tropicales presentan una gran cantidad de variantes morfológicas producto de la diversidad de condiciones generadas por la dinámica del dosel (Whitmore, 1984; Garwood, 1996). Características como la cobertura de la copa y la arquitectura son consecuencia, en gran medida, de los distintos procesos ontogénicos, mientras que parámetros de las hojas como el área foliar, el grosor de la lámina, el área foliar específica, entre otros son producto de las variaciones en la cantidad de luz (Sterck et al., 2006; Poorter, 1999). En el caso de M. zapota las variantes en la morfología de las plántulas se presentan bajo las mismas condiciones de dosel, situación que permite suponer que son producto de su ontogenia más que de respuestas de aclimatación a las variaciones en el dosel.

El desarrollo de hojas grandes en plántulas y árboles juveniles es un fenómeno que se ha descrito en diferentes especies de árboles tropicales, tanto tolerantes como

Cuadro 1. Características de las plántulas de Manilkara zapota localizadas en el sotobosque de una selva mediana subperennifolia de la región centro norte de Veracruz, México. Las plántulas están clasificadas con base en la longitud de su hoja más grande (LHMG). Se presentan valores promedio con su desviación estándar

Estado de la plántula con base en LHML (cm)

\begin{tabular}{|c|c|c|c|}
\hline Variables & $\begin{array}{c}\text { Hojas elipticas } \leq 5 \\
(n=153)\end{array}$ & $\begin{array}{l}\text { Hojas oblongas } 5.1-20 \\
(n=386)\end{array}$ & $\begin{array}{c}\text { Hojas oblongolanceoladas }>20 \\
(n=40)\end{array}$ \\
\hline Altura $(\mathrm{cm})$ & $9.80 \pm 1.98^{\mathrm{a}}$ & $12.68 \pm 2.75^{\mathrm{b}}$ & $25.6 \pm 5.16^{c}$ \\
\hline Número de hojas & $3.75 \pm 1.68^{\mathrm{a}}$ & $6.06 \pm 1.96^{\mathrm{b}}$ & $8.6 \pm 2.41^{\mathrm{c}}$ \\
\hline Cobertura $\left(\mathrm{cm}^{2}\right)$ & $43.03 \pm 24.6^{\mathrm{a}}$ & $191.28 \pm 165.17^{\mathrm{b}}$ & $1097 \pm 301.67^{c}$ \\
\hline Razón altura / LHMG & $2.42 \pm 0.97^{\mathrm{a}}$ & $1.53 \pm 0.35^{\mathrm{b}}$ & $1.03 \pm 0.22^{c}$ \\
\hline Área foliar específica $\left(\mathrm{cm}^{2} \mathrm{~kg}^{-1}\right)$ & $\begin{array}{c}16.19 \pm 3.5^{\mathrm{a}} \\
(\mathrm{n}=20)\end{array}$ & $\begin{array}{c}18.25 \pm 8.4^{\mathrm{a}} \\
(\mathrm{n}=10)\end{array}$ & $\begin{array}{c}11.56 \pm 0.90^{b} \\
(\mathrm{n}=10)\end{array}$ \\
\hline
\end{tabular}

Letras diferentes indican diferencias estadísticamente significativas $(t$-student, $\mathrm{p}<0.0001)$. 
intolerantes a la sombra. Se han hecho descripciones para especies pioneras de las selvas de Malasia (Whitmore, 1984) y México (Álvarez-Buylla y Martínez-Ramos, 1992) y para especies tolerantes a la sombra en bosques lluviosos semicálidos del sur de Japón (Kohyama, 1987), de selvas altas de Indonesia (Kohyama, 1991) y para las selvas de Malasia (Thomas e Ickes, 1995). En todos los casos se ha señalado que este proceso ofrece ventajas competitivas para el crecimiento bajo distintas condiciones del dosel, dado el incremento en la superficie fotosintética. Para M. zapota el incremento en el tamaño de las hojas tiene una relación directa y significativa con el incremento en los tiempos de supervivencia (Cruz-Rodríguez y LópezMata, 2004), situación que permite la conformación de un banco de plántulas persistente, de gran importancia para la dinámica poblacional de la especie (Cruz-Rodríguez et al., 2009). Una condición similar se presenta en el caso del árbol Chrysophyllum sp. nov. (Sapotacea), que es una especie dominante en el dosel de las selvas en Australia. Este árbol desarrolla plántulas grandes, con altas tasas de supervivencia y cuya dinámica explica la elevada abundancia de individuos adultos (Connell y Green, 2000).

Los valores de AFE de las plántulas de M. zapota presentan niveles bajos similares a los que presentan las plantas que se desarrollan en ambientes con condiciones limitantes (Poorter y de Jong, 1999). Sin embargo, resulta significativo que las plántulas con las hojas más grandes, con mayor área foliar, tengan los valores menores de AFE, ya que se ha señalado que en condiciones de baja irradiación, plántulas de diferentes especies de árboles tropicales, incrementan la intercepción de luz al aumentar la asignación de biomasa a las hojas; no obstante, producen hojas delgadas, con valores de AFE altos y con copas amplias para evitar el autosombreado. En contraste valores bajos de AFE se presentan en plántulas expuestas a luz intensa, con hojas pequeñas en las que se reduce la superficie de evapotranspiración (Poorter, 1999). Sin embargo, también se ha reportado que valores bajos de AFE permiten a las especies tolerantes a la sombra mantener sus hojas por largos periodos de tiempo y lograr un balance de carbón positivo, aunque la ganancia neta sea muy reducida (Myers y Kitajima, 2007; Montgomery y Chazdon, 2002). De hecho en las especies pioneras las hojas delgadas, con AFE alta, son muy productivas pero de corta duración y vulnerables a los herbívoros (Ackerly, 1997). En contraste, las hojas de las especies tolerantes a la sombra pueden tener una AFE baja, altos valores de biomasa seca y altas concentraciones de compuestos secundarios, los que les permite ser persistentes y longevas (Sterck et al., 1999).

El incremento en la cobertura de las plántulas conforme avanzan los estados ontogénicos, podría compensar el crecimiento vertical limitado que presentan las plántulas de M. zapota (Cruz-Rodríguez y López-Mata, 2004). Es posible que exista un compromiso entre el crecimiento en altura, para aumentar las probabilidades de alcanzar mejores oportunidades lumínicas en el futuro y el crecimiento horizontal para una asimilación efectiva en el presente (Kohyama, 1991).

¿Cómo se pueden formar hojas grandes y costosas bajo condiciones limitantes de luz y en individuos de bajo porte? Es probable que las plántulas pasen varios años acumulando recursos, tanto en tallo como en raíces y que los eventos de producción de hojas grandes se dan una vez que se ha alcanzado un cierto nivel de reservas. La presencia de una gran cantidad de gránulos de almidón almacenados en el tejido cortical y en parénquima xilemático de tallos (obs. pers.), indican que es necesario explorar esta idea, en virtud de que las reservas de carbohidratos son esenciales para la supervivencia de plántulas en el sotobosque, ya que con ellas enfrentan las pérdidas de carbono ocasionadas por la reducción en la luz y por el consumo de tejidos por herbívoros y la acción de patógenos (Myers y Kitajima, 2007).

\section{Literatura citada}

Agyeman, V. K., M. D Swaine y J. Thompson. 1999. Responses of tropical forest tree seedlings to irradiance and derivation of a light response index. Journal of Ecology 87:815-827.

Ackerly, D. 1997. Allocation, leaf display, and growth in fluctuating light environments. In Plant resource allocation, F. A. Bazzaz y J. Grace (eds.). Academic, San Diego, California. p. 234-264.

Álvarez-Buylla, E. y M. Martínez-Ramos. 1992. Demography and allometry of Cecropia obtusifolia, a neotropical pioneer tree-evaluation of the climax-pioneer paradigm for tropical rain forest. Journal of Ecology 80:275-90.

Baltzer, J. L. y S. C Thomas. 2007. Determinants of whole-plant light requeriments in Bornean forest tree saplings. Journal of Ecology 95:1208-1221.

Chazdon R. L. 1988. Sunflecks in the forest understory. Advances in Ecological Research 18:1-63.

Connell, J. H y P. T. Green. 2000. Seedling dynamics over thirtytwo years in a tropical rain forest tree. Ecology 81:568-584.

Cruz-Rodríguez, J. A. y L. López-Mata. 2004. Demography of the seedlings bank of Manilkara zapota (L.) Royen, in a subtropical rain forest of Mexico. Plant Ecology 172:227-235.

Cruz-Rodríguez, J. A., L. López-Mata y T. Valverde. 2009. A comparison of traditional elasticity and variance-standardized perturbation analyses: a case study with the tropical tree species Manilkara zapota (Sapotacea). Journal of Tropical Ecology 25:135-146.

Font Quer, P. 1977. Diccionario de Botánica. Labor, Barcelona. $1100 \mathrm{p}$.

Garwood, N. C. 1996. Functional morphology of tropical tree 
seedlings. In Ecology of tropical forest tree seedlings, M. D. Swaine (ed.). UNESCO/Parthenon, Paris/Carnforth. p. 59129.

Godínez-Ibarra, O. y L. López-Mata. 2002. Estructura, composición, riqueza y diversidad de árboles en tres muestras de selva mediana subperennifolia. Anales del Instituto de Biología, Universidad Nacional Autónoma de México, Serie Botánica 73:283-314.

Kitajima, K. 1994. Relative importance of photosynthetic traits and allocation patterns as correlates of seedling shade tolerance of 13 tropical trees. Oecologia 98:419-428.

Kohyama, T. 1987. Significance of architecture and allometry in saplings. Functional Ecology 1:399-404.

Kohyama, T. 1991. A functional model describing sapling growth under tropical forest canopy. Functional Ecology 53:83-90

Miquel, S. 1987. Morphologie fonctionnelle de plantules d'espèces forestières du Gabon. Bulletin du Muséum National d'Histoire Naturelle, 4e série, section B, Adansonia 9:101-121.

Miranda, F y E. Hernández-X. 1963. Los tipos de vegetación de México y su clasificación. Boletín de la Sociedad Botánica de México 28:29-179.

Montgomery, R. A. y R. L. Chazdon. 2002. Light gradient partitioning by tropical tree seedlings in the absence of canopy gaps. Oecologia 131:165-174.

Myers, J.A. y K. Kitajima. 2007. Carbohydrate storage enhances seedlings and stress tolerance in a neotropical forest. Journal of Ecology 95:383-395.

Ng, F. S. P. 1978. Strategies of establishment in Malayan foresters trees. In Tropical trees as living systems, P. B. Tomlinson y M. H. Zimmermann (eds.). Cambridge University Press. p. 129-162.

Pennington, T. D. 1992. Sapotacea. Flora Neotropica 52: 1-771. Pennington, T. D. y J. Sarukhán. 1998. Los árboles tropicales de México. Universidad Nacional Autónoma de México/Fondo de Cultura Económica, México, D.F. 413 p.

Poorter, H. y R. de Jong. 1999. A comparison of specific leaf area, chemical composition and leaf construction cost of field plants from 15 habitats differing in productivity. New Phytologist 143:163-176.

Poorter, L. 1999. Growth responses of rain-forest tree species to a light gradient: the relative importance of morphological and physiological traits. Functional Ecology 13:396-410.

Rijkers, S.A., P. J. de Vries, T. L. Pons y F. Bongers. 2000. Photosyntetics induction insaplings of the three shadetolerant tree species: camparing understorey and gap habitats in French Guiana rain Forest. Oecologia 125:331-340.

Rozendaal, D. M. A., V. H Hurtado y L. Poorter, L. 2006. Plasticity in leaf of 38 tropical tree species in response to light; relations with light demand and adult stature. Functional Ecology 20:207-216.

Sterck, F. J., D. B. Clark, D. A. Clark, y F. Bongers. 1999. Light fluctuations, crown traits, and response delays for tree saplings in a Costa Rica lowland rain forest. Journal of Tropical Ecology 15:83-95.

Sterck, F. J., L. Poorter y F. Schieving. 2006. Leaf traits determine the growth-survival trade-off across rain forest tree species. The American Naturalist 167:758-765.

Sterck, F., M. Martínez-Ramos, G. Dyer-Leal, J. RodríguezVelázquez. y L. Poorter. 2003. The consequences of crown traits for the growth and survival of tree saplings in a Mexican lowland rainforest. Functional Ecology 17:194-200.

Thomas. S. C. y K. Ickes. 1995. Ontogenetic changes in leaf size in Malaysian rain forest trees. Biotropica 27:427-434.

Turner, I. M. 1990. Tree seedling growth and survival in a Malaysian rain forest. Biotropica 22: 146-154.

Whitmore, T. C. 1984. Tropical rain forest of the Far East, second edition. Oxford Science.

Wilson, P. J., K. Thompson y J. G. Hodgson. 1999. Specific leaf area and dry matter content as alternative predictors of plant strategies. New Phytologist 143:155-162. 